\title{
High voltage and current metrology at NIST. Precision measurements of the past, present, and the future.
}

\author{
G.J. FitzPatrick and E.D. Simmon \\ National Institute of Standards and Technology, Gaithersburg, MD, USA
}

\begin{abstract}
The accurate measurement of high voltages and currents is vital to monitoring power flows in electric power systems, metering electric power and energy, and, of course, to characterizing the behavior of electrical insulation. NIST has been making precision electrical measurements since its inception a century ago. This paper discusses metrology that is now used or under development at NIST for ac and pulse high voltages and currents. The compensated current comparator is at the heart of most precision ac measurements made at NIST because it offers the greatest sensitivity and lowest measurement uncertainty even today, some forty years after its development. It is used for characterizing ac high voltage and current transformers in terms of their ratio and phase angles, and also for capacitor ratio measurements. Precision ac high voltage dividers with active electronic compensation have also been recently developed. Measurement systems utilizing the magneto-optic Faraday effect are now being developed for both ac and pulsed currents. The basic principles of these devices and systems, their practical limitations, and measurement uncertainties are discussed in this paper.
\end{abstract}

\section{Introduction}

The National Institute of Standards and Technology (NIST) is now in its centennial year and has been conducting research in electrical measurements for most of that time. Some of the earliest measurement services provided by NIST's predecessor, the National Bureau of Standards (NBS), were for tests of voltage and current transformers used as reference standards by electric utilities. An example of an NBS certificate for a voltage transformer test performed in 1929 is shown in Figure 1. Magnetic instrument transformers are still used for monitoring and metering of electricity in the electric power grid, but the techniques used for testing this equipment have been improved over the years resulting in a reduction in the measurement uncertainties. In addition to the conventional voltage and current transformers, new technologies have been developed that utilize optical techniques to measure voltages and currents in electric power systems. These technologies offer important advantages over purely electrical instruments: their all-dielectric construction makes them

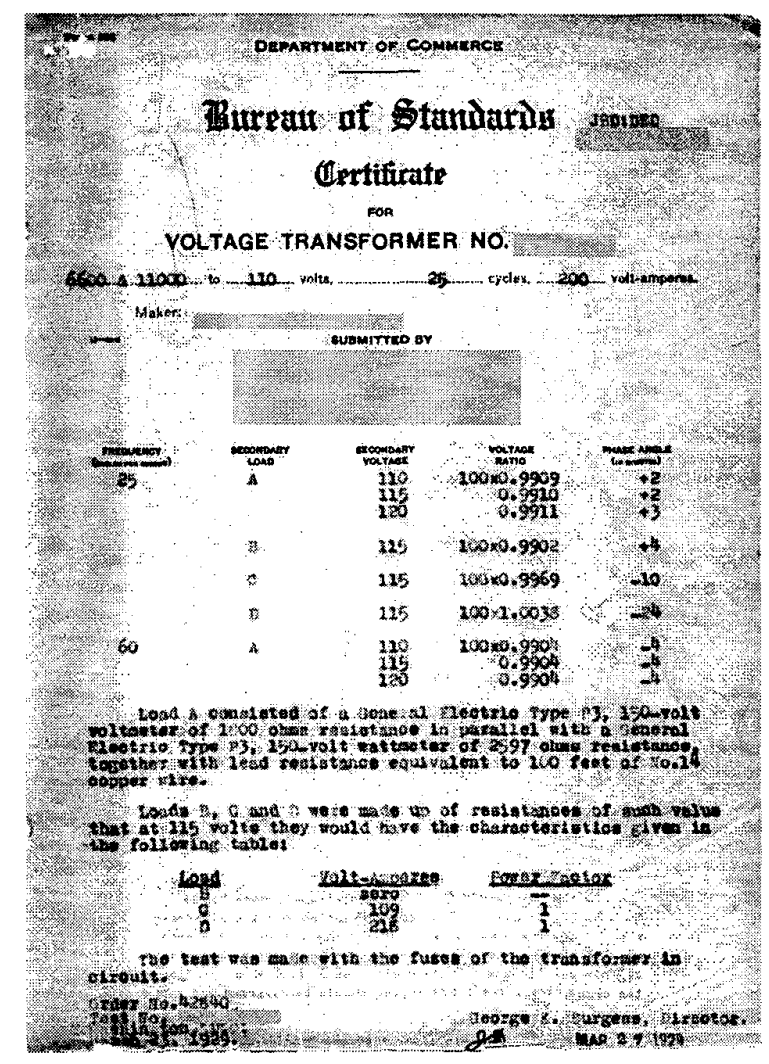

Figure 1: National Bureau of Standards test certificate. The certificate was issued for a test of a voltage transformer performed in 1929.

much better suited for high voltage applications than magnetic transformers that require large volumes of insulation. The significantly broader bandwidth of optical sensors makes it possible to use the same sensor for metering, protective relaying, and diagnostics.

This techniques used at NIST for the testing and calibration of high voltage capacitors and transformers, precision techniques for high voltage measurements, and calibration methods for the optical technologies now being employed in electric power systems are described in the following sections. 


\section{High Voltage Capacitors and Transformers}

The NIST system used for high voltage ac measurements is described in [1]. The heart of the measurement system is a specialized three-winding transformer known as a current comparator[2], which is shown schematically in Figure 2. Two of the windings on the comparator are wound on the core so that the resultant magnetic fluxes oppose each other when the currents in the windings are in phase. By adjusting the ratio of the windings, the fluxes can be made to nearly cancel each other so that the residual flux detected by the third winding, $N_{\mathrm{D}}$, is minimized. The equations that describe flux balance are

and

$$
N_{\mathrm{x}} I_{\mathrm{x}} \approx N_{\mathrm{s}} I_{\mathrm{s}}
$$

$$
\frac{I_{\mathrm{x}}}{I_{\mathrm{s}}} \approx \frac{N_{\mathrm{s}}}{N_{\mathrm{x}}},
$$

so that at balance the ratio of the currents are determined by the known ratio of the turns in the comparator windings. The turns ratio for high quality comparators are known with errors of the order of one turn in $10^{7}[1]$. The compensated current comparator circuit is significantly more complex than indicated in Figure 2. It includes additional active circuitry for balance of quadrature currents and for compensation of errors that arise due to winding and lead resistance. With this additional circuitry, the ratio of and phase angle between two capacitors shown in Figure 3 can be measured and the capacitance of a test capacitor is determined from the value of the standard capacitance

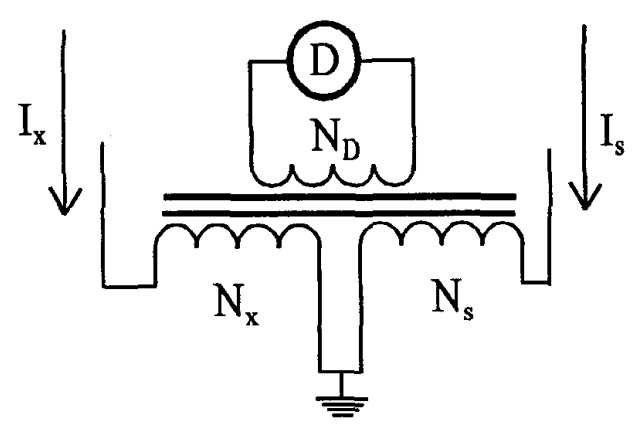

Figure 2. The basic current comparator circuit. The fluxes in the core due to currents $I_{\mathrm{x}}$ and $I_{s}$ are balanced by varying the $N_{\mathrm{x}}$ and $N_{\mathrm{s}}$ windings until the voltage measured across the $N_{\mathrm{D}}$ winding by the detector $D$ is minimized.

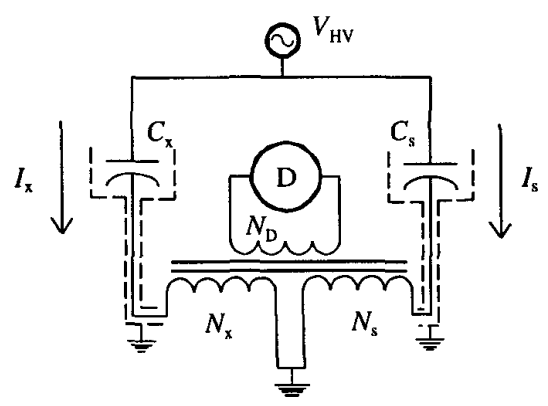

Figure 3: Capacitance ratio determination. The unknown capacitor, $C_{\mathrm{x}}$, and the standard capacitor, $C_{\mathrm{s}}$, are connected in parallel to the high voltage source, $V_{\mathrm{Hv}}$. At balance, the capacitance ratio is given by $C_{\mathrm{x}} / C_{\mathrm{s}}=N_{\mathrm{s}} / N_{\mathrm{x}}[1]$.

through the balance equation given in [1]:

$$
C_{\mathrm{x}}=\left(N_{\mathrm{s}} / N_{\mathrm{x}}\right) C_{\mathrm{s}}
$$

The ratio and phase angle for a voltage transformer are deteremined using the setup of Figure 4 , where the primary and secondary windings are connected to the reference capacitors $C_{\mathrm{HV}}$ and $C_{\mathrm{LV}}$. At balance the transformer ratio is determined by the turns ratio and the ratio of reference capacitors [1]:

$$
V_{\mathrm{HV}} / V_{\mathrm{LV}}=\left(N_{\mathrm{s}} / N_{\mathrm{x}}\right)^{\prime}\left(C_{\mathrm{s}} / C_{\mathrm{x}}\right)
$$

Typical uncertainties for capacitors and voltage transformers determined with the NIST measurement

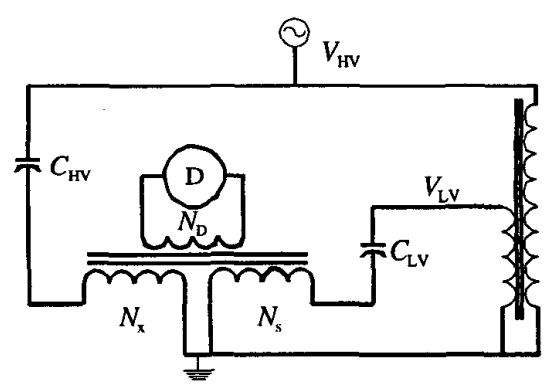

Figure 4: Voltage transformer ratio measurement. The primary winding of the voltage transformer under test is connected in parallel with the standard high voltage capacitor, $C_{\mathrm{HV}}$ to the source high voltage, $V_{\mathrm{HV}}$. The secondary winding is connected to the standard low voltage capacitor, $C_{\mathrm{LV}}$. At balance the voltage transformer ratio is given by $V_{\mathrm{HV}} / V_{\mathrm{LV}}=\left(N_{\mathrm{s}} / N_{\mathrm{x}}\right)^{\prime}\left(C_{s} / C_{\mathrm{x}}\right)[1]$ 
Table 1: Typical measurement uncertainties for capacitors and voltage transformers[3].

\begin{tabular}{|c|c|c|}
\hline \multirow{2}{*}{ Capacitors } & Capacitance & $10 \mu \mathrm{F} / \mathrm{F}$ \\
\cline { 2 - 3 } & Phase Angle & $10^{-5}$ radian \\
\hline $\begin{array}{c}\text { Voltage } \\
\text { Transformers }\end{array}$ & Ratio & $300 \mu \mathrm{V} / \mathrm{V}$ \\
\cline { 2 - 3 } & Phase Angle & $\begin{array}{c}3 \times 10^{-4} \\
\text { radian }\end{array}$ \\
\hline
\end{tabular}

system are given in Table I. The best uncertainties achievable for capacitance and transformer ratio can be an order of magnitude smaller.

\section{Precision AC High Voltage Measurements}

Many high voltage laboratories have gas-filled, high voltage (HV) capacitors that can be that can be used for precision ac HV measurements. For this application, the the HV capacitor is used as the high voltage arm of an active voltage divider. In an active divider developed at NIST, special emphasis was placed upon obtaining a low phase angle uncertainty as required for measurement of load losses of power transformers. One advancement over an earlier related development [4] was to use low-voltage capacitors, thus eliminating the need for a second high-voltage capacitor element.

The principle of the divider described in [5] uses the concept of a controlled voltage source, phaselocked to the input HV signal, that is inserted into the low-voltage arm of the divider to add to the amplifier output as shown if Figure 5. The voltage of the source is adjusted to the value

$$
V_{1}\left(C_{1} / C_{2}\right)(1-d)
$$

where $d$ is a small deviation from the desired value, $V_{1}$ is the input high voltage, $C_{1}$ is value of the $\mathrm{HV}$ capacitor, and $C_{2}$ is the value of the low-voltage capacitor. The equation for the voltage ratio of this circuit becomes

$$
\left(V_{2} / V_{1}\right)=-\left(C_{1} / C_{2}\right)(1-d / G),
$$

where $G$ is the open loop gain of the amplifier. Since $d$ is adjusted to be smaller than 0.001 and $G$ is larger than 1000 , the difference between the ideal voltage ratio of the divider and the capacitor ratio can be less than $10^{-6}$. The NIST divider, which is used as a reference standard in voltage divider testing, has uncertainties in divider ratio of less than $20 \mu \mathrm{V} / \mathrm{V}$ and phase angle uncertainties are lower than $2 \times 10^{-6}[5]$. For laboratories having a

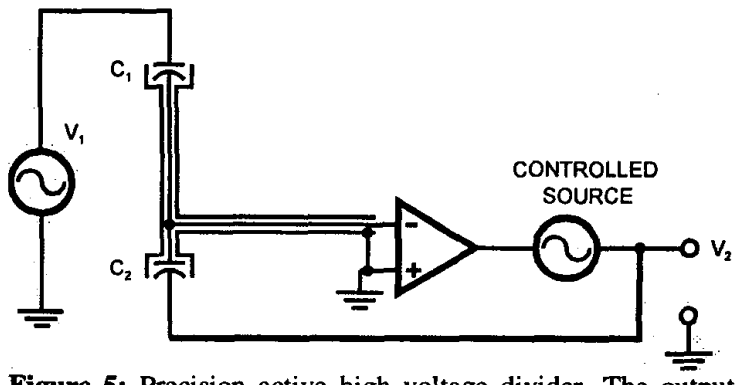

Figure 5: Precision active high voltage divider. The output voltage given by $V_{2} \approx-\left(C_{1} / C_{2}\right) V_{1}$ is only an approximation because of the finite gain of the operational amplifier. This accuracy limitation is overcome through insertion of a controlled source in the low-voltage arm of the circuit.

good quality high voltage capacitor, a precision ac measurement system can be built using relatively inexpensive off-the-shelf components.

\section{Optical Current Sensors}

Analog methods for providing calibrations for conventional CTs have been well developed for many years[6]. However the commercialization of optical current transducers (OCTs) has required development of new precision calibration techniques. These transducers measure a high current input and provide a low power output, often $10 \mathrm{~V}$ or less[7]. OCTs have important advantages over their conventional CT counterparts, such as an inherently wider bandwidth. Some optical sensors are capable of measuring from dc to $\mathrm{MHz}$ frequencies. The same sensor therefore can be used with different electronics for measuring direct current, power frequency current, and current transients. Since these sensors are typically made of optical fiber or bulk glass, their all-dielectric construction is appealing for high voltage applications.

Techniques developed for OCTs having analog voltage outputs are applicable to OCTs having digital outputs as well. The measurement system shown in Figure 6 is used to test OCTs designed for power frequency metering applications, for which the uncertainty requirements are usually the most demanding. Simultaneous measurements are made of the outputs of both the OCT and the CT/shunt combination with precision multimeters used in their digitizer modes. Once the digital waveforms are obtained, they are analyzed using a nonlinear leastsquares curve-fitting routine to find the best-fit parameters of the sinusoidal output waveforms. Using the known reference CT/shunt ratio and phase angle, the OCT transimpedance ratio and phase is determined. 


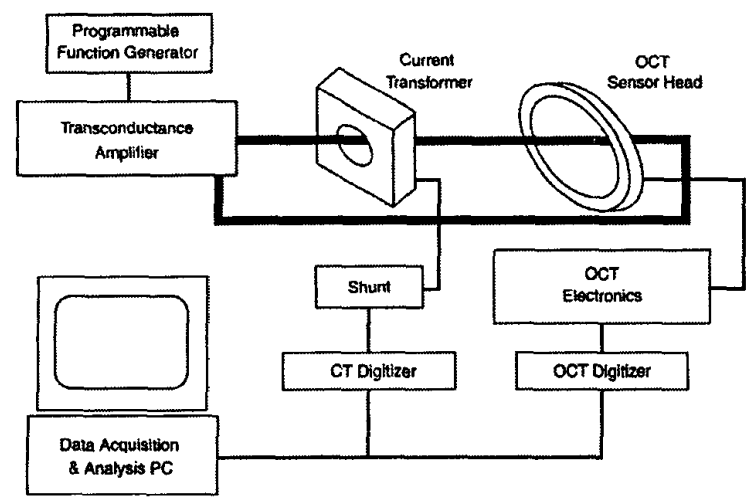

Figure 6: Simplified optical current transducer (OCT) test setup. The OCT is placed in series with a reference current transformer (CT) having a shunted output. The voltage output of the OCT and the CT shunt voltage are digitized and compared to determine the OCT transimpedance.

The total uncertainty for OCT transimpedances determined with this method is estimated to be about $160 \times 10^{-6}(0.016 \%)$ for currents between $10 \%$ and $100 \%$ of the OCT rated currents. The corresponding uncertainty for phase angle is $260 \times 10^{-6}$.

One advantage of OCTs over CTs for power system applications is their wide dynamic range, which is needed for metering of independent power generators that are now coming on line under electric utility deregulation. These generators may have large power outputs when running at full capacity, but consume power at a much lower level when operating in "housekeeping" mode. Two or more CTs may be needed to accurately over the range from amperes to kiloamperes, whereas a single OCT may have the desired linearity to cover the entire current range with the desired uncertainty. The signal-to-noise ratio of the OCT output can be low when the current level falls below $10 \%$ of the OCT current rating. The calibration techniques described here require further development to verify linearity at these low current levels.

\section{The Future}

NIST and its predecessor, NBS, have performed research in electrical measurements almost since the birth of electric power industry. NIST continues to use the current-comparator based measurement systems to provide calibrations for conventional voltage transformers used in power systems and capacitors used in high voltage laboratories. Improved measurement techniques have been developed to provide costeffective ways for measuring high voltages with low uncertainties. As new technologies, such as OCTs, are created, NIST will continue efforts to develop new techniques to provide the measurements required for their reliable use in electric power sytems and high voltage applications.

\section{References}

[1] E.D. Simmon, G.J. FitzPatrick, O. Petersons, "User's Guide To The NIST Compensated Current Comparator For High-Voltage Applications", NIST Technical Note, TNXXXX, 2001

[2] W.J.M. Moore and P.N. Miljanic, The Current Comparator, London, UK: Peter Peregrinus, Ltd., 1988

[3] J.L. Marshall, Ed., "NIST Calibration Services Users Guide 1998", Natl. Inst. Of Stand. And Technol. (U.S.), NIST Special Publication 250, January 1998.

[4] O. Petersons and S. P. Mehta, "An active voltage divider and phase shifter," IEEE Trans. on Instrum. and Meas., vol. IM-36, pp. 362-368, 1987.

[5] O. Petersons, G.J. FitzPatrick, E.D. Simmon, "An active highvoltage divider with $20 \mu \mathrm{V} / \mathrm{V}$ uncertainty", IEEE Trans. On Instrum. and Meas., vol. 46, no. 2, pp. 430-434, April, 1997

[6] J.D. Ramboz and O. Petersons, "A Calibration Service for Current Transformers," Natl. Inst. of Stand. and Technol. (U.S.) NIST Special Publication 250-36, June 1991.

[7] Emerging Technology Working Group and Fiber Optic Sensors Working Group, "Optical transducers for power systems - a review," IEEE Transactions on Power Delivery, vol. 9, no. 4, pp. 1778-1788, October 1994.

Author addresses: Gerald J. FitzPatrick, National Institute of Standards and Technology, 100 Bureau Drive, Mail Stop 8113, Gaithersburg, MD, USA 208998113, Email: gerald.fitzpatrick@nist.gov.

Eric D. Simmon, National Institute of Standards and Technology, 100 Bureau Drive, Mail Stop 8113, Gaithersburg, MD, USA 20899-8113, Email: eric.simmon@nist.gov. 